\title{
Vein Patterns as Bio-Metric Identifier using Euclidean Distance
}

\author{
Sunita Aeri and Sukhvinder Kaur \\ SDDIET, Barwala, Haryana, India \\ SDDIET, Barwala, Haryana, India
}

\begin{abstract}
Hand Vein patterns are one of the safest biometric information due to their strong resilience against the impostor attacks. In the presented work, a new approach for biometric authentication using infrared thermal hand vein patterns is proposed. The proposed work presents a Euclidean distance based vein's pattern based biometric authentication that can be used for ascertaining the biometric identity of person under scanner. The vein patterns are grabbed using the infra red (IR) thermal cameras and after applying some image pre-processing operations, a binary image is obtained consisting of veins crossings and intersections. The binary image is thinned using the morphological operations and a single line thinned image pattern is obtained. The thinned image pattern is now examined for intersections extractions and inter-distance between intersections. The inter-distance between intersections of vein patterns are stored in a data base. Further, when a test vein pattern is brought under test, the data base information is compared to that of the test pattern using Euclidean distances. Minimum the Euclidean distance, more is the equivalency of the test pattern to data base pattern
\end{abstract}

\section{KEYWORDS}

Region of Interest, Vein Bio-metrics, Segmentation, Thresholding, Imposter Attacks, Image Thinning

\section{INTRODUCTION}

Biometrics are automated methods of recognizing a person based on a physiological or behavioral characteristic. Among the features measured are; face, fingerprints, hand geometry, handwriting, iris, retinal, vein, and voice. Biometric systems are superior because they provide a nontransferable means of identifying people not just cards or badges. The key point about an identification method that is "nontransferable" means it cannot be given or lent to another individual so nobody can get around the system - they personally have to go through the control point.

Hand vein patterns are the vast network of blood vessels under-neath a person's skin. The vein patterns are unique individual and are stable over a long period of time It is invisible to human eye that way its avoid the external distortion and it is not easy to replicate the vein patterns as compared to other biometric traits. Other skin attributes like, color, moles, hair, etc, make it

\section{RELATED WORKS}

In this paper vascular pattern of a palm is used as a biometric identification of a person. There is no chance of leakage of secret information because veins are inner part of human being. Blood vessel pattern are used as an identity of a person in "palm vein authentication system". This technology provides high level security information, as it is compared with other techniques used previously. Palm vein pattern authentication technique used in many banks in Japan .Fujitsu Company trying further to increase the speed of verification by improving sensor capacity [1].
In customer's mobile device, important information may get corrupted so that easy methods are adapted and implemented to avoid such types of leakage. Biometric methods such as" Real Time Recognition system" using finger vein is adapted in this paper which provide high level of accuracy to avoid chances of leakage of important personal information [2].

Blood vessel patterns acts as a biometric identification of individual, but blood have near infrared light absorption properties that's why contact less sensors are used .veins images are unstable \& noisy. A new method is adapted for encoding purpose .Spatial information \& chain codes are used for feature extraction of individual persons [3].

A nonlinear method of KPCA is used to reduce the 2D images into 1D image vector also decrease dimensional values in Palm -Dorsa vein recognition based on Kernel Principal analysis shows that the algorithm used in it is very useful \& there is less error in comparison KPCA+FLD with PCA+FLD .This process is more error free which is $97.63 \%$ \& accuracy rate is $5.26 \%[4]$.

Word biometric authentication means how a person personal behave $\&$ his mantle attitude .In this paper, vascular pattern of palm is taken, zoom image of vascular pattern is passes through infrared light which then processed by many stages1.)This infrared image is converted into gray image ii.)Gray image is converted into binary image by algorithm. Vascular pattern thinner algorithm has maximum level of security [5].

In recent few years child abuse cases are increases day by day .Many criminals blackmail children by taking images of their sexual activities with children .Vascular patterns identification for forensic investigation were very impossible, new method of optical based vein uncovering (OBVU) is light sensitive used for forensic investigation, but algorithm for this method is not proposed yet. A new method is adopted to overcome this problem, color optimization scheme is used in which automatic matching algorithm for vein identification is developed .This automatic matching algorithm for vein identification is used over 300 colors pairs \& its performance is much better than OBVU for perpetrators \& victim identity[6].

For security purpose biometric genuine method is best suited for this purpose. We take thermal image of back of hand or we can say we obtain vein pattern of back of hand by using IR web camera. This thermal image is then goes through four different phases -image capturing, image preprocessing, feature extraction \& genuine module. In this technique ,IR web camera is attached with doer, whenever another person open the camera capture the vein image of back of hand. By Housdroff distance technique, both the images matched, the system automatically finds the difference between vein patterns by line segment [7]. 
Template important

\begin{tabular}{|l|l|l|}
\hline $\mathrm{P}_{8}$ & $\mathrm{P}_{1}$ & $\mathrm{P}_{2}$ \\
\hline $\mathrm{P}_{7}$ & $\mathrm{P}_{0}$ & $\mathrm{P}_{3}$ \\
\hline $\mathrm{P}_{6}$ & $\mathrm{P}_{5}$ & $\mathrm{P}_{4}$ \\
\hline
\end{tabular}

security

is

more

authentication.

Biometric systems are suffered from various warnings. In this paper we study about various attacks that biometric system suffered. For this some algorithms are produced .In this biometric system is combined with cryptography which converts Dorsal vein template into novel cryptographic structure which are fuzzy vault .Some steps are involved into Dorsal hand vein images for enhancement, smoothing \&compression. Some steps are i.) Thinning ii.) Binary encoding iii.) Feature extraction, then it will generate Fuzzy Vaults. for decoding purpose biometric template is formed \& combined with Fuzzy vaults to produce the required output .Finally FNMR \& FMR values are calculated with/without noise .[8].

In analysis of palm vein pattern recognition algorithms \& system, palm vein has high degree of security as palm veins are internal part of body .Nobody can change or take it away. Algorithms explain: detection of region of interest (ROI), segmentation, feature extraction \& matching. All processes are carried out in a machine learning techniques without any error [9].

A multimodal biometric system based on finger vein \& hand vein recognition, a new scheme is proposed which is called multimodal in which both finger vein $\&$ hand vein image are combined which gives high degree of performances .Monogenic Local Binary Pattern (MLBP) used for finger vein identity \& improved Gaussian matched filter (IGMF)is used for hand vein identity. Area under curve is near to unity. [10].

Unimodal method of analysis of finger veins \& finger prints creates many disadvantages. In multimodal method of analysis of finger veins \& finger prints combination provides high degree of accuracy, where finger veins \& finger prints point intercept each other are points of identity of a person. These multimodals biometric are hoax to any attacks. It provides better security at lesser cost \& lesser error rate [11].

Veins are internal part of a body so there are no chances of delicacy .Finger veins, back of hand \& palm has a complex vascular pattern as compared to unimodal biometric which has some disadvantages like performances based. Multimodal authentication technique including fusion of palm veins $\&$ face biometric provide high degree of accuracy \& security identification [12].

Multimodal system overcomes the limitation exists in unimodal biometric system .Finger prints \& finger veins are most popular because of much accuracy \& security \&lesser cost. This research work completed in five parts I.)Finger print \& finger vein acquisition ii.)Preprocessing iii.) Feature extraction IV.) Feature matching v.)Fusion. It provides high degree of accuracy \& output. It increased performance [13]

In this paper finger vein identification procedure adapted this method Personalized Best Bit Map(PBBM).This method worked upon binary pattern ,in which best suited bit is selected for matching after that algorithm is generated ,finger vein frame work prepared .It follows some more steps like : i.) preprocessing ii.)Feature extraction iii.)Matching .PBBM provide good performance high reliability. PBBM used as a framework [14].

\section{METHODOLOGY}

Vein patterns images are acquired using the infra red (IR) cameras. The acquired images are blur in nature as they are the thermal signature of the veins pattern. The grabbed images are normally in jpeg format and are converted to gray scale format using the rgb2gray command in matlab. The veins image patterns are enhanced using the histogram equalization method where the histogram of the vein patterns are modified in order to enhance the vein patterns from the back ground.

The histogram equalized image (O/P Img) is given by:

$$
\mathrm{O} / \mathrm{P} \operatorname{Img}(\mathrm{x}, \mathrm{y})=\frac{\operatorname{CDF}(0)-\operatorname{CDF}(\min )}{\operatorname{MxN}-1} \cdot(L-1)
$$

Where $\operatorname{CDF}(\mathrm{i}), \mathrm{CDF}(\mathrm{min})$ are the $\mathrm{i}_{\text {th }}$ gray level and minimum cumulative distribution function. $(\mathrm{M}, \mathrm{N})$ is the image size and $\mathrm{L}$ is the maximum gray level intensity in image.

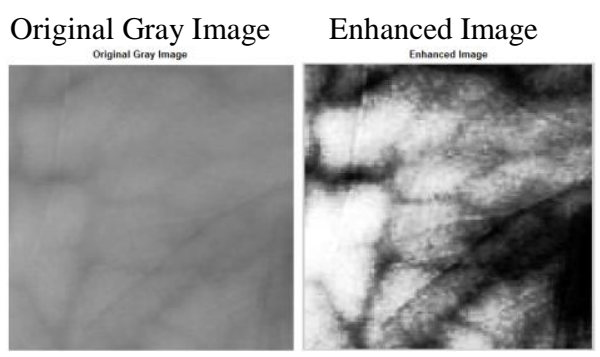

The enhanced images are now binarized using the Otsu algorithm. Otsu algorithm is based on selecting a threshold value based on minimum within class variance method. Minimum within class variance is computed for all gray level values i.e. from $\mathrm{i}=0$ to 255 and for which gray level, the minimum class variance is minimum, that gray level is used as the threshold value. Based on the computed threshold value, the image is binarized i.e. the gray value less than threshold value are converted to back ground and above are converted to pattern.

The binary pattern so obtained consists of salt and pepper type noise. It is removed by using the following algorithm:

If $(\mathrm{P} 0=\mathrm{BLACK}) \& \mathrm{P} 1=\mathrm{P} 2=\mathrm{P} 3=\mathrm{P} 4=\mathrm{P} 5 \mathrm{P} 6=\mathrm{P} 7=\mathrm{P} 8=$ WHITE)

Then $\mathrm{P} 0$ is the Background Pixel.

If $(\mathrm{P} 0=$ WHITE) \& $\mathrm{P} 1=\mathrm{P} 2=\mathrm{P} 3=\mathrm{P} 4=\mathrm{P} 5 \mathrm{P} 6=\mathrm{P} 7=\mathrm{P} 8=$ BLACK)

Then P0 is the Object Pixel.

Finally the binary images so obtained are now thinned to single pixel width vein patterns. This is done by using the pixel neighborhood connectivity algorithm and 8-neighbors of a pixel under scanner. This can be easily implemented using bwmorph command in matlab. 
The intersections are extracted by using the following masks:

\begin{tabular}{|l|l|l|}
\hline 1 & 0 & 1 \\
\hline 0 & 1 & 0 \\
\hline 0 & 0 & 0 \\
\hline
\end{tabular}

\begin{tabular}{|l|l|l|}
\hline 1 & 0 & 0 \\
\hline 0 & 1 & 0 \\
\hline 1 & 0 & 0 \\
\hline
\end{tabular}

\begin{tabular}{|l|l|l|}
\hline & 0 & 0 \\
\hline 0 & 1 & 0 \\
\hline 1 & 0 & 1 \\
\hline
\end{tabular}

\begin{tabular}{|l|l|l|}
\hline 0 & 0 & 1 \\
\hline 0 & 1 & 0 \\
\hline 0 & 0 & 1 \\
\hline
\end{tabular}

\begin{tabular}{|l|l|l|}
\hline 1 & 0 & 1 \\
\hline 0 & 1 & 0 \\
\hline 1 & 0 & 1 \\
\hline
\end{tabular}

\begin{tabular}{|l|l|l|}
\hline 0 & 1 & 0 \\
\hline 1 & 1 & 1 \\
\hline 0 & 1 & 0 \\
\hline
\end{tabular}

The central pixel is the pixel under scanner and the coordinates of the central pixel are taken as the intersection points. Following images show the intersections obtained in a template image.

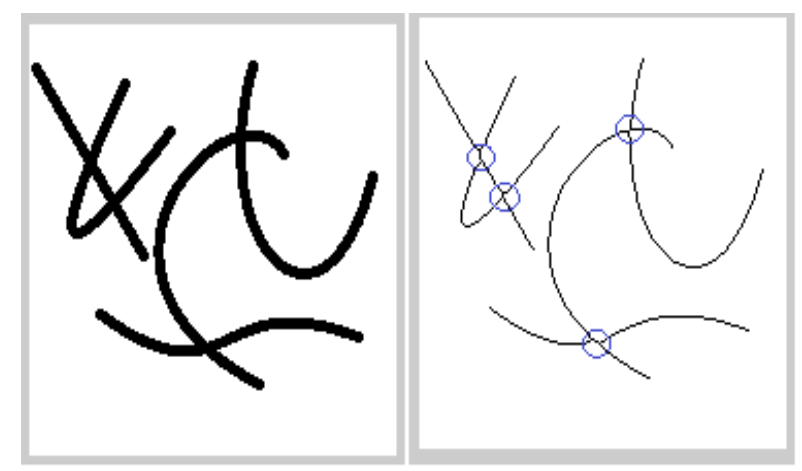

The inter-distances between intersections are computed by the following formula:

Say $\left(\mathrm{x}_{1}, \mathrm{y}_{1}\right)$ and $\left(\mathrm{x}_{2}, \mathrm{y}_{2}\right)$ are coordinates of two intersections, then the inter-distance between them is given by:

$$
D=\sqrt{ }\left(\mathbf{x}_{1}-\mathbf{x}_{2}\right)^{2}+\left(\mathbf{y}_{1}-\mathbf{y}_{2}\right)^{2}
$$

The inter-distances are now stored in a data base along with veins pattern attributes like name and physical identity.

Same steps are repeated for other vein patterns and a data base is generated. Further when a test vein pattern is examined, same steps are repeated and inter-distances are computed. Now the Euclidean distances between test pattern and data base patterns are computed by using the following formula:

$$
\mathrm{ED}=\sqrt{ }\left(\mathbf{D}_{\mathrm{T}}-\mathbf{D}_{\mathrm{db}}\right)^{\mathbf{2}}
$$

Where $D_{t}$ and $D_{d b}$ are the test and data base vein pattern interdistances. Sort the ED vector for minimum ED. The vein pattern in data base with minimum ED is the equivalent vein pattern to test vein image.

\section{FLOW CHART}
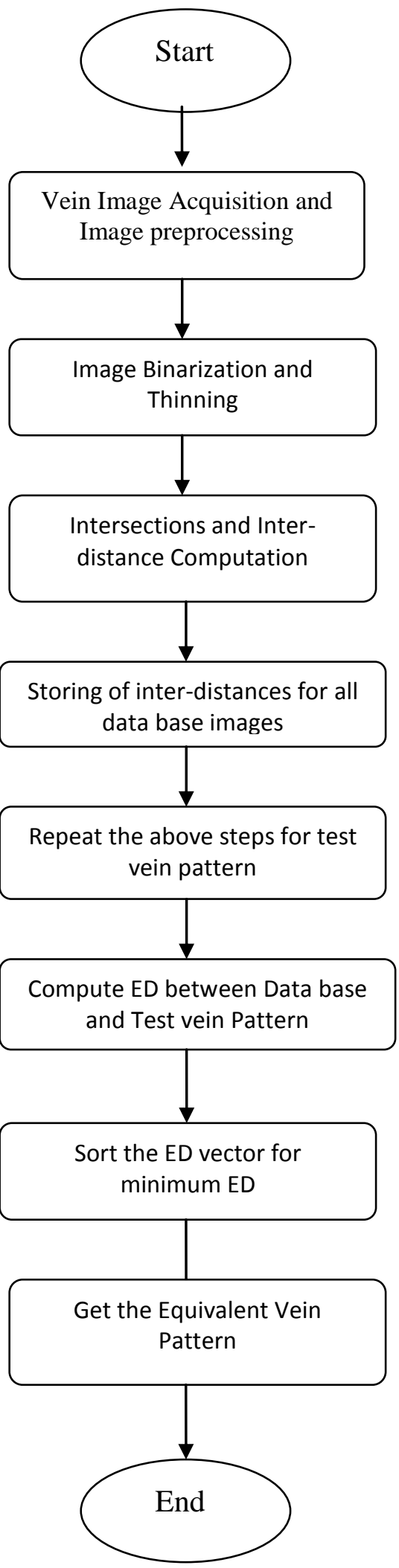


\section{RESULTS AND CONCLUSION}

A data base of 100 vein patterns is created. The proposed work has been implemented on approximately 100 veins patterns. The inter-distance between test pattern and data base image pattern shows a good results based on Euclidean distance. And a fair identification of vein to that of person identity is obtained. The added advantage with vein feature is that the vein pattern can not be changed at any time or by any means. We have achieved a $90 \%$ accuracy in identifying the persons based on vein patterns. Further, it should be noted that the vein pattern biometric is complementary to the other bio-metric information and should not be taken as the only source of biometric identity. Basically vein complements to the other biometric identity.

\section{REFERENCES}

[1] Ishani Sarkar, Farkhod Alisherov, Tai-hoon Kim, and Debnath Bhattacharyya, "Palm Vein Authentication System: A Review", International Journal of Control and Automation Vol. 3, No. 1, March,

[2] Vanathi G, Nigarihaa R, Uma Maheswari G \& Sujitha R, "Real Time Recognition System Using Finger-Vein", International Journal of Advanced Electrical and Electronics Engineering (IJAEEE), 2278-8948, Volume2, Issue-2, 2013

[3] The International Journal of Advanced Electrical and Electronics Engineering (IJAEEE), Vanathi G, Nigarihaa $\mathrm{R}$, Uma Maheswari G \& Sujitha R Electronics and Communication Engineering, Avinashilingam University Coimbatore, India E-mail:ganesh.vanathi@gmail.com, nigarihaa22@gmail.com,umaguruswamy24@gmail.com, sujitharjrm@gmail.com

[4] Anika Pug1, Daniel Hartung2 and Christoph Busch1;2anika.pflug@cased.dedaniel.hartung@hig.nochr istoph.busch@hig.no1 University of Applied Sciences Darmstadt - CASED, Haardtring 100,64295 Darmstadt, Germanyhttp://www.h-da.de2 Norwegian Information Security Laboratory (NISlab)H_gskolen i Gj_vik, Teknologivn. 22,2815 Gj_vik, Norwayhttp://www.hig.no

[5] Journal of Computational Information Systems 8: 4 (2012) 1545-1552Available at http://www.Jofcis.comJing LIU 1;2; Dingyu XUE2, Jianjiang CUI 2, Xu JIA 21College of Information and Engineering, Shenyang University of Chemical Technology, Shenyang110142, China2School of Information Science and Engineering, Northeastern University, Shenyang 110004, China

[6] Researchers P. Ghosh, R. Dutta Department of Computer Science and Engineering Department of Electronics and Communication Engineering Surendra Institute of Engg. \& Management, WBUT, Siliguri, India Email-_ papri.mss@gmail.com, ritam_siliguri@yahoo.com 1 | (C) I J A I T I 20012 VOLUME 1 NUMBER 2 (Mar/Apr 2012)

[7] 6. 978-1-4673-1228-8/12/\$31.00 @2012 IEEEHengyi Zhang, Chaoying Tang, Adams Wai-Kin Kong and Noah CraftSchool of Computer Engineering, Nanyang Technological University, Block N4, Nanyang Avenue, Singapore 639798Los Angeles Biomedical Research Institute at Harbor-UCLA Medical Center, 1124 West Carson St, Torrance, CA 90502
[8] INFO Computer Engineering ISSN: 2249-3980 \& EISSN: 2249-3999, Volume 2, Issue 1, 2012, pp.-1921. Available online

at http://www.bioinfo.in/contents.php?id=322PAL M.M.1 AND JASUTKAR R.W.2 $1 \mathrm{M}$. Tech (ESC), I.T DEPT.(S.D.C.E ), MS, India.2M. Tech (VLSI), G.H Raisoni College of Engineering, MS, India. *Corresponding Author: EmailMadhuridec9@gmail.com, rwjasutkar@gmail.com

Brindha, J Biomet Biostat 2012, 3:4 V Evelyn Brindha* Department of Computer Science and Engineering, Bannari Amman Institute of Technology, Sathyamangalam, Erode, Tamil Nadu, India

[9] ISSN 2321-9017 Volume 1, No.1, June - July 2013

International Journal of Bio-Medical Informatics and e-Health Available Online

at http://warse.org/pdfs/ijbmieh02112013.pdfMona A Ahmed1, Hala M. Ebied2,El-Sayed M. El-Horbaty3, Abdel-Badeeh M. Salem41, 3, 4Computer Science Department2Scientific Computing DepartmentFaculty of Computer and Information SciencesAin Shams University, Cairo, Egypt eng.monaa_cs@hotmail.com, halam@cis.asu.edu.eg,shorbaty@cis.asu.edu.eg, absalem@cis.asu.edu.eg

[10] Randa Boukhris Trabelsi et.al / International Journal of Engineering and Technology (IJET). Randa Boukhris Trabelsi\#1, Alima Damak Masmoudi\#2, and Dorra Sellami Masmoudi\#3

\#Computer Imaging and Electronics Systems group (CIELS) from Control and Energy ManagementLaboratory(CEMLab), Sfax Engineering School, Tunisia, BP W, 3038 Sfax, Tunisia, Phone: (216-74)274.088, Fax. (21674)275.595.1_trabelsiboukhrisranda@live.fr 2damak_alima@yahoo.fr 3dorra.masmoudi@enis.rnu.tn

[11] Proceedings of the 4th National Conference; INDIACom-2010 Computing For Nation Development, February 25 - 26, 2010 Bharati Vidyapeeth's Institute of Computer Applications and Management, New Delhi Arunkumar.V, Malathy.C Computer science Department, SRM UNIVERSITY, SRM Nagar, Kattankulathur603203, Kancheepuram District, TamilNadu, India arunkumr1990@gmail.com, malathy_c@yahoo.com

[12] IJACSA) International Journal of Advanced Computer Science and Applications, Vol. 4, No S.F.Bahgat College of computers and IT Taif University Taif, KSA S. Ghoniemy College of computers and IT TaifUniversity Taif, KSA M. Alotaibi College of computers and IT Taif University Taif, KSA

[13] International Journal of Computer Applications (0975 8887) Volume 51- No.5, August 2012 T.Sheeba, Muscat College, P.O.Box:2910, P.C:112, Ruwi, Sultanate of Oman M.Justin Bernard, W.J. Towell Engineering, P.O.BOX:1040, P.C:112, Ruwi, Sultanate of Oman

[15] Sensors 2012, 12, 1738-1757; doi: 10.3390/s120201738sensorsISSN 14248220www.mdpi.com/journal/sensors, Gongping Yang, Xiaoming $\mathrm{Xi}$ and Yilong Yin * School of Computer Science and Technology, Shandong University, Jinan 
250101, China;E-Mails: gpyang@sdu.edu.cn (G.Y.); fyzq10@126.com (X.X.)

* Author to whom correspondence should be addressed; E-Mail: ylyin@sdu.edu.cn;Tel.: +86-531-8839-1367; Fax: +86-531-8839-1367.

\section{Author Profile}

The author ${ }^{1}$ is pursuing her M.Tech. in ECE from SDDIET, Barwala affiliated to KU, Kurukshetra. Her field of interest is in image processing based system design and applications. 